\title{
Scheduling Suspendable, Pipelined Tasks with Non-Preemptive Sections in Soft Real-Time Multiprocessor Systems*
}

\author{
Cong Liu and James H. Anderson \\ Department of Computer Science, University of North Carolina at Chapel Hill
}

\begin{abstract}
While most prior work on multiprocessor real-time scheduling focuses on independent tasks, dependencies due to non-preemptive sections, suspensions, and pipelinebased precedence constraints are common in practice. In this paper, such complexities are considered in the context of the global earliest-deadline-first scheduling algorithm. It is shown that any periodic task system with such dependencies can be transformed into one with only suspensions in a way that preserves maximum per-task response times. This result enables analysis directed at systems with suspensions to be applied if non-preemptive sections and/or pipelines are present as well.
\end{abstract}

\section{Introduction}

The growing prevalence of multicore platforms has led to much recent work on multiprocessor real-time scheduling. Most of this work has been directed at scheduling problems that arise when systems of independent tasks are to be supported. In practice, however, programming methodologies are often used that result in dependencies among tasks. As multiprocessor platforms become more ubiquitous, and real-time applications of greater complexity are supported on them, it is crucial that scheduling-related research be extended so that such dependencies can be properly addressed. In this paper, we consider this issue in the context of periodic task systems in which task dependencies may exist due to non-preemptive sections, suspensions, and pipeline-based precedence constraints.

Any one of these kinds of dependencies can cause a task system to be difficult to analyze from a schedulability perspective. For instance, non-preemptive sections may cause scheduling anomalies (e.g., shortening a job's execution time may actually increase some job's response time) and suspensions may cause unbounded job response times even in lightly-loaded systems [9]. Still, situations may exist in which all three kinds of dependencies are present. Consider, for example, a pipelined real-time computation where some tasks may require disk accesses and non-preemptivity arises due to system calls or critical sections. The timing correct-

*Work supported by AT\&T, IBM, and Sun Corps.; NSF grants CNS 0834270 and CNS 0834132; ARO grant W911NF-09-1-0535; and AFOSR grant FA9550-09-1-0549. ness of such a system may be quite difficult to analyze, particularly if deadline misses cannot be tolerated. However, we show in this paper that the situation is not nearly so bleak, if bounded deadline tardiness is acceptable.

Bounded tardiness is a notion that has been studied extensively in the context of global scheduling algorithms, and such algorithms are our focus as well. In global algorithms, tasks are scheduled from a single run queue and may migrate across processors. Such algorithms stand in contrast to partitioning algorithms, which statically assign tasks to processors and use per-processor run queues. Under partitioning schemes, constraints on overall utilization are required to ensure timeliness even if bounded deadline tardiness can be tolerated. On the other hand, a variety of globalscheduling approaches are capable of ensuring bounded tardiness in ordinary periodic and sporadic systems (without suspending tasks or pipeline tasks) with no utilization loss, even if non-preemptive sections exist $[2,6]$. In recent research, this work has been extended to show that, in fullypreemptive systems, bounded tardiness can be ensured if tasks either suspend [9] or have pipeline constraints [7, 8] (but not both), provided certain utilization restrictions hold.

In this paper, we consider whether these research results can be combined. That is, we address the problem of deriving conditions under which bounded tardiness can be ensured when all of the above-mentioned behaviorsnon-preemptive sections, pipelines, and suspensions-are allowed. In considering this problem, we focus specifically on the global earliest-deadline-first (GEDF) algorithm, but our analysis could potentially be extended to apply to other global algorithms as well. Our main result is a transformation process that converts any implicit-deadline periodic task system with suspensions, pipelines, and nonpreemptive sections into a simpler system with only suspensions. In the simpler system, each task's maximum job response time is at least that of the original system. This result allows tardiness bounds to be established by focusing only on the impacts of suspensions. While this result was motivated by our interest in tardiness bounds, the transformation we present is also relevant to hard real-time systems.

Related Work. To our knowledge, the problem addressed in this paper has not been considered before, in the context of either global or partitioned scheduling. However, some work has been done in which the effects of non- 
preemptive sections, pipelines, or suspensions have been considered independently, as noted above [2, 6-9]. Prior work on pipelining has also been done in the context of distributed systems (which must be scheduled by partitioning approaches) [3, 4, 13].

In work pertaining to uniprocessors (and by extension multiprocessors scheduled via partitioning), several sufficient schedulability tests have been presented for analyzing tasks with suspensions [1, 5, 10-12, 14]. Intractability/impossibility results pertaining to be suspensionoriented analysis have also been obtained $[15,16]$.

Contributions. In this paper, we consider periodic task systems with suspensions, pipelines, and non-preemptive sections; for conciseness, we henceforth refer to these systems as NPS systems. We show how to transform such a task system into a simpler periodic task system with only suspensions. In the transformed system, per-task maximum job response times are at least those of the original system. We show that this enables prior results on systems with suspensions [9] to be applied to derive tardiness bounds for more complex systems, as scheduled by GEDF. Such bounds are applicable to NPS task systems provided certain utilization constraints are met. We analyze the loss of system capacity inherent in these constraints via an extensive experimental study involving randomly-generated task systems. This study shows that the capacity loss is moderate to non-existent in scenarios where the executions costs of pipeline stages vary moderately and suspension and nonpreemptive-section lengths are moderate.

Organization. The rest of this paper is organized as follows. Sec. 2 describes our system model. The transformation discussed above is obtained via a sequence of subtransformations, which are described in Secs. 3 and 4. In Sec. 5, a tardiness bound for periodic NPS task systems is derived. The above-mentioned experimental evaluation is then presented in Sec. 6. Sec. 7 concludes.

\section{NPS Task Systems}

We consider the problem of scheduling a set $\tau^{N P S}=$ $\left\{T_{1}, \ldots, T_{n}\right\}$ of $n$ periodic pipeline tasks on $m \geq 2$ identical processors, where any such task may suspend and contain non-preemptive sections. Below, we present definitions pertaining to pipeline tasks. These definitions are illustrated with an example given later.

An $h$-stage pipeline task $T_{l}$, where $1 \leq h \leq m$, consists of $h$ subtasks, $T_{l}^{1}, \ldots T_{l}^{h}$. Each subtask is released repeatedly, with each such invocation called a job. Jobs alternate between computation and suspension phases. We assume that each job of $T_{l}^{h}$ executes for at most $e_{l}^{h}$ time units (across all of its computation phases), suspends for at most $s_{l}^{h}$ time units (across all of its suspension phases), and has at most $c_{l}^{h}$ computation phases. We place no restric- tions on how these phases interleave (a job can even begin or end with a suspension phase). We also do not restrict their lengths, other than upper-bounding them. Note, in particular, that 0-length computation phases are allowed in our model. The circumstances under which a 0-length computation phase can commence execution are just like with any other computation phase (as described later). However, a 0-length computation phase that commences execution at time $t$ finishes execution at time $t$. Computation phases are also allowed to contain non-preemptive code segments. The maximum duration of any non-preemptive segment of $T_{l}^{h}$ is denoted $b_{l}^{h}$, and the maximum such value taken over all subtasks in $\tau^{N P S}$ is denoted $b_{\max }$.

The $j^{t h}$ job of $T_{l}^{h}$, denoted $T_{l, j}^{h}$, is released at time $r_{l, j}^{h}$ and has a deadline at time $d_{l, j}^{h}$. Associated with each pipeline task $T_{l}$ is a period $p_{l}$, which specifies both the exact time between two consecutive job releases of $T_{l}$ and the relative deadline of each such job, i.e., $d_{l, j}^{h}=r_{l, j}^{h}+p_{l}$. The release time of job $T_{l, j}^{h}$ is required to satisfy $r_{l, j}^{h}=$ $(j-1+h-1) \cdot p_{l}$. The utilization of subtask $T_{l}^{h}$ is defined as $u_{l}^{h}=e_{l}^{h} / p_{l}$, and the utilization of $T_{l}$ is defined as $u_{l}=\sum_{i=1}^{h} u_{l}^{i}$. The utilization of the task system $\tau^{N P S}$ is defined as $U_{\text {sum }}=\sum_{T_{l} \in \tau^{N P S}} u_{l}$. Notice that, if $h=1$, and for the subtask $T_{l}^{h}, s_{l}^{h}=0$ holds, then $T_{l}^{h}$ is an ordinary (non-suspending) periodic task.

Successive jobs of the same subtask are required to execute in sequence. Also, for $h>1$, job $T_{l, j}^{h}$ cannot commence execution (no matter whether its first phase is a computation phase or a suspension phase) until the last phase of the prior-stage job $T_{l, j}^{h-1}$ (be it a computation phase or suspension phase) completes. (That is, the $j^{\text {th }}$ per-stage jobs must execute in sequence.) To avoid confusion when discussing these precedence constraints, we will refer to $T_{l, j-1}^{h}$ as the $L$-predecessor of $T_{l, j}^{h}$ (assuming $j>1$ ), and $T_{l, j}^{h-1}$ as the $U$-predecessor of $T_{l, j}^{h}$ (assuming $h>1$ ). ("L" and "U" stand for "left" and "upper", respectively).

The timeliness constraint considered in this paper is that deadline tardiness be bounded. If a job $T_{i, j}^{k}$ completes at time $t$, then its response time is defined as $t-r_{i, j}^{k}$ and its tardiness is defined as $\max \left(0, t-d_{i, j}^{k}\right)$. A pipeline task's tardiness is the maximum of the tardiness of any job of any of its subtasks. Note that, when a job of a subtask misses its deadline, the release time of the next job of that subtask is not altered. Despite this, it is still required that a job cannot execute in parallel with either of its predecessors.

The results of this paper pertain to the GEDF scheduling algorithm. Under GEDF, released jobs are prioritized by their deadlines. Jobs in $\tau^{N P S}$ are ordered based on their priorities: $T_{i, v}^{w} \prec T_{a, b}^{c}$ if and only if $d_{i, v}^{w}<d_{a, b}^{c}$ or $\left(d_{i, v}^{w}=d_{a, b}^{c}\right) \wedge(i=a) \wedge(w<c)$ or $\left(d_{i, v}^{w}=d_{a, b}^{c}\right) \wedge(i<a)$. Thus, when comparing equal deadlines, the tie is broken in favor of earlier stages of the same pipeline task, and any 


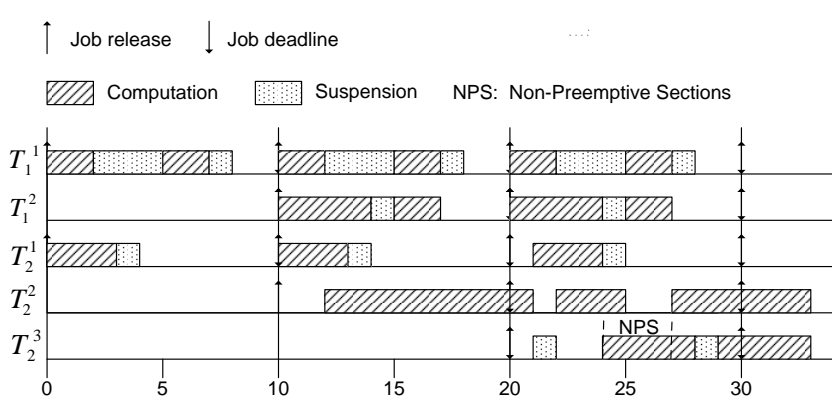

Figure 1: Example NPS task system.

remaining tie is broken by task ID. $T_{i, v}^{w}$ has higher priority than $T_{a, b}^{c}$ if and only if $T_{i, v}^{w} \prec T_{a, b}^{c}$. At any time, GEDF schedules the highest-priority enabled jobs (the term "enabled" is formally defined in Sec. 3) on the available processors subject to the constraint that a job in a non-preemptive section cannot be preempted. GEDF becomes fully preemptive when $b_{\max }=0$ and fully non-preemptive when $b_{l}^{h}=e_{l}^{h}$, for all $l, h$.

Example 1. Fig. 1 depicts an example GEDF schedule of a suspendable pipelined task system with non-preemptive sections, scheduled on three processors. This task system contains a two-stage pipeline task $T_{1}$ with a period of 10 time units and a three-stage pipeline task $T_{2}$ with a period of 10 time units. $T_{1}^{1}$ executes for 2 time units, then suspends for 3 time units, executes for another 2 time units, and finally suspends for 1 time unit. $T_{1}^{2}$ executes for 4 time units, then suspends for 1 time unit, and executes for another 2 time units. $T_{2}^{1}$ executes for 3 time units, and then suspends for 1 time unit. $T_{2}^{2}$ executes for 9 time units. $T_{2}^{3}$ first suspends for 1 time unit, then executes non-preemptively for 3 time units and then preemptively for another time unit, suspends for 1 time unit, and finally executes for 4 time units. As seen in the GEDF schedule, $T_{2,1}^{2}$ misses its deadline at time 20 by 1 time unit, which causes $T_{2,1}^{3}$ to start its first suspension phase at time 21 . At time $24, T_{2,1}^{3}$ starts executing its first computation phase since $T_{1,3}^{1}, T_{1,2}^{2}, T_{2,3}^{1}$ are suspended at that time. Since this execution is non-preemptive, at time $25, T_{1,2}^{2}$ preempts $T_{2,1}^{2}$ instead of $T_{2,1}^{3}$.

Roadmap. In this paper, we show how to transform $\tau^{N P S}$, a periodic NPS task system, into a periodic task system with only suspensions. This transformation requires two steps:

1. Transform $\tau^{N P S}$ into $\tau^{P S}$, where $\tau^{P S}$ denotes a fully preemptive suspendable pipelined task system, by treating blocking times due to non-preemptive sections as suspensions. This is dealt with in Sec. 3 .

2. Transform $\tau^{P S}$ into $\tau^{S}$, where $\tau^{S}$ is a periodic task system with only suspensions (i.e., it contains no pipeline tasks and it is fully preemptive), by treating pipeline

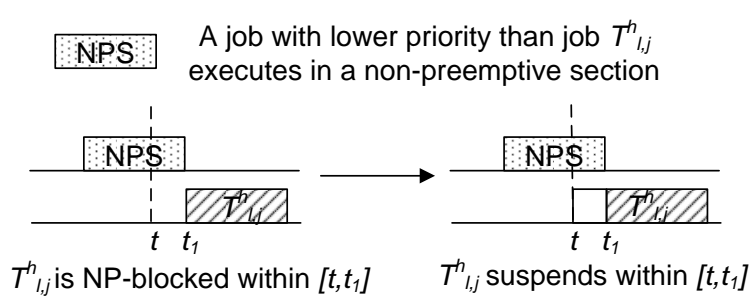

Figure 2: Modeling NP-blocking as suspension.

blocking times (see Sec. 4) as suspensions. This is dealt with in Sec. 4.

\section{Transforming $\tau^{N P S}$ to $\tau^{P S}$}

We transform $\tau^{N P S}$ into $\tau^{P S}$ by treating blocking times due to non-preemptive sections as suspensions.

Definition 1. We say that a task system $\tau$ is concrete if the actual execution cost and suspension time of every job of each task is fixed. For any $\tau$ ( $\tau$ may be any of the task systems mentioned in the roadmap at the end of the prior section), we let $\bar{\tau}$ denote any arbitrary concrete instantiation of it.

Definition 2. A job $T_{l, j}^{h}$ is enabled if it has been released and both its U-predecessor (if any) and L-predecessor (if any) have completed. A job is considered to be completed if it has finished its last phase (be it suspension or computation).

A job $T_{l, j}^{h}$ is non-preemptively blocked, or NP-blocked, at time $t$ if it is among $m$ highest-priority enabled jobs according to GEDF, but it cannot execute because lowerpriority jobs are executing non-preemptively at $t$. This can happen only when $T_{l, j}^{h}$ commences executing one of its computation phases. Given that any job $T_{l, j}^{h}$ has at most $c_{l}^{h}$ such phases and the maximum length of any job's nonpreemptive section is at most $b_{\max }$, we have the following lemma.

Lemma 1. Any job $T_{l, j}^{h}$ in $\tau^{N P S}$ can be NP-blocked for at most $c_{l}^{h} \cdot b_{\text {max }}$ time units.

Given Lemma 1, we can define $\tau^{P S}$ by simply treating NP-blocking times as suspensions. That is, we view all nonpreemptive sections as preemptive, and for any subtask $T_{l}^{h}$, we increase $s_{l}^{h}$ by $c_{l}^{h} \cdot b_{\max }$, which by Lemma 1 upperbounds the NP-blocking time of $T_{l}^{h}$. This is illustrated in Fig. 2. The theorem below immediately follows.

Theorem 1. For any concrete instantiation $\bar{\tau}^{N P S}$ of $\tau^{N P S}$, there exists a concrete instantiation $\bar{\tau}^{P S}$ of $\tau^{P S}$ such that $\bar{\tau}^{N P S}$ and $\bar{\tau}^{P S}$ have equivalent GEDF schedules. ${ }^{1}$

\footnotetext{
${ }^{1}$ That is, if $S^{N P S}\left(S^{P S}\right)$ is the GEDF schedule for $\bar{\tau}^{N P S}\left(\bar{\tau}^{P S}\right)$, then job
} 
Note that this transformation strongly exploits the fact that, in our task model, suspension phases are upperbounded, and hence, can be reduced to reflect actual NPblocking times. Note also that the "reverse" of this theorem may not hold: for a concrete instantiation $\bar{\tau}^{P S}$ of $\tau^{P S}$, there may not exist a concrete instantiation $\bar{\tau}^{N P S}$ of $\tau^{N P S}$ such that $\bar{\tau}^{P S}$ and $\bar{\tau}^{N P S}$ have equivalent GEDF schedules.

Corollary 1. For any subtask $T_{l}^{h}$, if the maximum response time of any job of $T_{l}^{h}$ in any GEDF schedule for $\tau^{P S}$ is $z$ time units, then the maximum response time of any such job in any GEDF schedule for $\tau^{N P S}$ is at most $z$ time units.

\section{Transforming $\tau^{P S}$ to $\tau^{S}$}

In this section, we transform $\tau^{P S}$ into $\tau^{S}$ by treating pipeline blockings as suspensions. Notice that $\tau^{S}$ is a periodic task system where the first job of any task is released at time 0 . However, according to our pipeline task model as described in Sec. 2, the first job of any non-first-stage subtask $\left(T_{l}^{h}\right)$ (where $h \geq 2$ ) is released at time $(h-1) \cdot p_{l}$. However, we can easily add some "extra" initial jobs to each non-firststage subtask so that each subtask starts at time 0. Specifically, for any subtask $\left(T_{l}^{h}\right)$ (where $h \geq 2$ ), we add $h-1$ such "extra" jobs, $T_{l, 2-h}^{h}, T_{l, 2-h+1}^{h}, \ldots, T_{l,-1}^{h}, T_{l, 0}^{h}$, with execution and suspension times of zero. Adding these jobs will not affect the schedule.

As shown in [7], the fundamental problem that makes pipeline scheduling difficult is PL-blocking, as defined below.

Definition 3. If a released job's L-predecessor has completed, but its U-predecessor has not, then it is said to be $P L$ blocked. Note that a first-stage job cannot be PL-blocked because it has no U-predecessor. Note also that the first job of any subtask cannot be PL-blocked because it has no Lpredecessor.

PL-blocking is illustrated in Fig. 3(a). Note that, in the figure, we use $J$ to denote job $T_{i, v}^{k}, J_{U}$ to denote $J$ 's Upredecessor $T_{i, v}^{k-1}$ (if $k \geq 2$ ), and $J_{L}$ to denote $J$ 's Lpredecessor $T_{i, v-1}^{k}$ (if $v \geq 2$ ). This shorthand notation is sometimes used in the discussion that follows.

If no job of any subtask of any pipeline task is PLblocked, then every job's U-predecessor completes no later than its L-predecessor. In this case, the precedence constraint enforced by the periodic task model, which requires consecutive jobs of the same subtask to execute in sequence, is sufficient to ensure that the pipeline task is scheduled correctly. Therefore, it is the PL-blocking effect that makes pipeline task scheduling different from ordinary periodic task scheduling. (Note that, when jobs are never tardy, PLblocking cannot happen.)

$T_{l, j}^{h}$ is scheduled at time $t$ in $S^{N P S}$ if and only if it is scheduled at time $t$ in $S^{P S}$. A similar interpretation of "equivalent" applies to Theorem 2. (a)

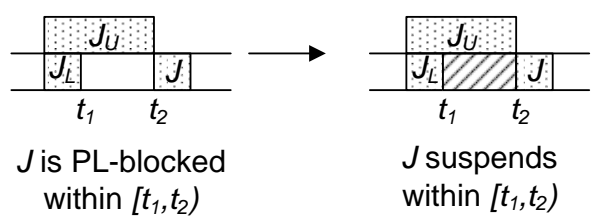

(b)

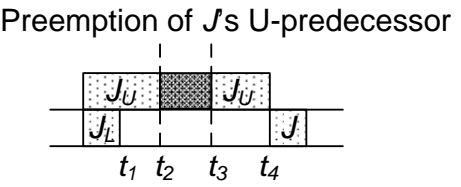

$J$ 's $U$-predecessor is preempted within $\left[t_{2}, t_{3}\right)$, and $J$ is PL-blocked within $\left[t_{1}, t_{4}\right)$
Figure 3: (a) Job $J$ is PL-blocked at time $t$. (b) Treating PL-blockings as suspensions.

Based upon the above observation, we intend to transform pipeline tasks into suspending tasks by treating PLblocking times as suspensions, and eliminating precedence constraints among pipeline stages. The transformation converts an $h$-stage pipeline task into $h$ independent suspending tasks. If a job is PL-blocked within the time interval $\left[t_{1}, t_{2}\right)$, then it could be deemed to suspend within $\left[t_{1}, t_{2}\right)$, as illustrated in Fig. 3(a). The new system obtained by performing such a transformation may have schedules in which the eliminated precedence constraint is violated. However, each schedule of the old system is also a valid schedule of the new system, so any per-(sub)task response time bound established for the new system is applicable to the old system as well.

In order to treat PL-blockings as suspensions, we need to upper-bound a job's PL-blocking time. However, preemptions (as defined below) may cause difficulties in upperbounding PL-blocking times.

Definition 4. If job $T_{i, v}^{w}$ is enabled at time $t$ and does not suspend at $t$, but does not execute at $t$, then it is preempted at $t$. The total time for which $T_{i, v}^{w}$ is preempted is called its preemption time.

Fig. 3(b) illustrates why preemption is a problem. Here, $J$ is PL-blocked within $\left[t_{1}, t_{4}\right)$ and its U-predecessor is preempted within $\left[t_{2}, t_{3}\right)$. Since it is difficult to upper-bound preemption times, it is also difficult to upper-bound PLblocking times. The situation is made even more complicated by the fact that "upstream" jobs in the pipeline must also be considered. These jobs are characterized as follows.

Definition 5. We define the set of $U$-jobs of any job $T_{i, v}^{w}$ to be $T_{i, v}^{w-1}, T_{i, v}^{w-2}, \ldots, T_{i, v}^{1}$.

A U-job of $T_{i, v}^{w}$ is a job of the same pipeline task that may impact the scheduling of $T_{i, v}^{w}$, directly or indirectly, through precedence constraints. 
MV The U-predecessor of J's U-predecessor (a $U$-job of $J$ )

Preemption of one of J's U-jobs

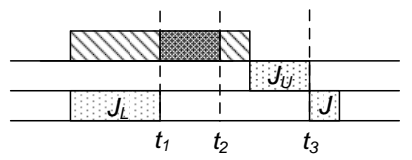

One of J's U-jobs is preempted within

$\left[\mathrm{t}_{1}, \mathrm{t}_{2}\right)$, and $\mathrm{J}$ is PL-blocked within $\left[\mathrm{t}_{1}, \mathrm{t}_{3}\right)$

(a)

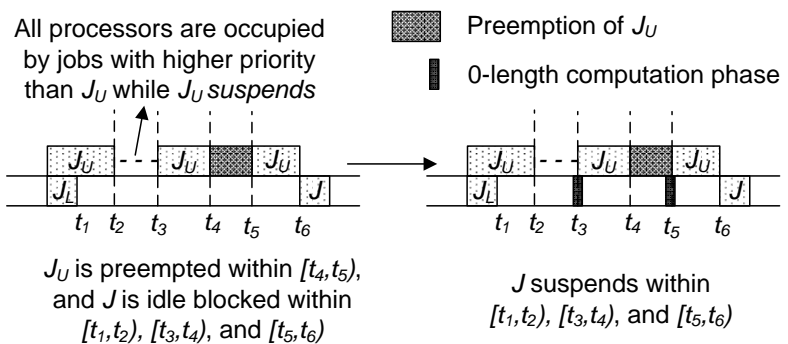

(b)

Figure 4: (a) The preemption of a U-job of $J$ may increase its PL-blocking time. (b) Treating idle blocking time as suspensions.

The preemption of any $\mathrm{U}$-job of $J$ may increase $J$ 's PLblocking time. Consider, for example, Fig. 4(a). Here, one of $J$ 's U-jobs (other than $J_{U}$ ) is preempted within $\left[t_{1}, t_{2}\right.$ ), which causes $J$ 's PL-blocking time to be $t_{3}-t_{1}$.

Fortunately, although preemption causes problems in upper-bounding PL-blocking times, it is only necessary to upper-bound idle blocking times, as defined below, in order to transform a pipeline task into independent suspending tasks. This is because, if one of $T_{i, v}^{w}$ 's U-jobs is preempted at time $t$ while $T_{i, v}^{w}$ is blocked, then there can be no idle processors at $t$. Hence, it is not necessary to consider $T_{i, v}^{w}$ to be suspended at that time.

Definition 6. If $T_{i, v}^{w}$ is PL-blocked at time $t$, and at least one processor is idle or occupied by a job with lower priority than $T_{i, v}^{w}$, then $T_{i, v}^{w}$ is idle blocked at $t$. The total time for which $T_{i, v}^{w}$ is idle blocked within a time interval $\left[t, t^{\prime}\right)$ is called its idle blocking time within $\left[t, t^{\prime}\right)$.

To see that it is only necessary to treat a job's total idle blocking time as suspensions, consider Fig. 4(b). As seen in the figure, we can consider $J$ to suspend within $\left[t_{1}, t_{2}\right)$, and then execute a 0-length computation phase, which must be scheduled by GEDF at some $t^{\prime}$ within $\left[t_{3}, t_{6}\right)$, where $t_{6}$ is the first time at which $J$ is scheduled. $\left(t^{\prime}=t_{3}\right.$ in Fig. 4(b).) If $t^{\prime}<t_{4}$, then $J$ can be defined to suspend within $\left[t^{\prime}, t_{4}\right)$. Then $J$ executes another 0-length computation phase, which must be scheduled by GEDF at some $t^{\prime \prime}$ within $\left[t^{\prime \prime}, t_{6}\right)$. ( $t^{\prime \prime}=t_{5}$ in Fig. 4(b).) Finally $J$ can then be defined to suspend within $\left[t_{5}, t_{6}\right)$. Note that the total needed suspension length is upper-bounded by the total idle-blocking time of $J$. The same is true of more complicated scenarios. (Later, we consider the possibility of adding some of the needed suspension time to account for $J$ 's idle blocking to $J_{L}$ instead of $J$.)

To treat idle blocking times as suspensions, an upper bound on the total idle blocking time of a job must be determined. This is dealt with in Lemma 2 below.

Definition 7. If $T_{i, v}^{w}$ 's first phase is an execution (suspension) phase and it begins executing (a suspension) for the first time at $t$, then $t$ is called its start time, denoted $S\left(T_{i, v}^{w}\right)$. If $T_{i, v}^{w}$ 's last phase (be it execution or suspension) completes at time $t^{\prime}$, then $t^{\prime}$ is called its finish time, denoted $F\left(T_{i, v}^{w}\right)$. Let $E T\left(T_{i, v}^{w}\right)$ denote the first time instant when when $T_{i, v}^{w}$ is enabled.

Lemma 2. The total idle blocking time of any job $T_{i, v}^{k}$ in $\tau^{P S}$ is at most $\sum_{j=1}^{j=k-1}\left(e_{i}^{j}+s_{i}^{j}\right)$.

Proof. We prove the claim by induction on $k$. The base case is trivial: $T_{i, v}^{1}$ has no U-predecessor and thus is not PL-blocked. We shall now prove the induction step, $k>1$. Note that if $J_{L}$ does not exist, then $J$ is released at time 0 and will not be blocked, by Def. 3. So, assume that $J_{L}$ exists. For simplicity, let $J_{U L}$ denote $T_{i, v-2}^{k-1}$, and $J_{U U}$ denote $T_{i, v-2}^{k-2}$, as shown in Fig. 5 (a). Let $e_{U}=e_{i}^{k-1}$, $e_{L}=e_{i}^{k}, s_{U}=s_{i}^{k-1}$, and $s_{L}=s_{i}^{k}$, and let $t=S\left(J_{L}\right)$ and $t^{\prime}=F\left(J_{L}\right)$. Also let $e_{U}^{\prime}\left(e_{L}^{\prime}\right)$ denote the actual run-time execution time of $J_{U}\left(J_{L}\right)$. Note that if $J$ is not released at or before $t^{\prime}$, then $J$ is not blocked at $t^{\prime}$, but may be after $t^{\prime}$, in which case $J$ 's idle blocking time may not be maximal. Thus, it suffices to assume that $J$ is released at or before $t^{\prime}$.

Case 1: $J_{U U}$ is complete at $t$ (or, it does not exist), as shown in Fig. 5 (b). Observe that $J_{U L}$ is complete by time $t$ (otherwise, $J_{L}$ could not execute). Thus, by the condition of Case $1, J_{U}$ is enabled at or before $t$, which implies that all U-jobs of $J$ other than $J_{U}$ complete at or before $t$. If $J_{U}$ completes by time $t^{\prime}$, then $J$ is not PL-blocked, so assume otherwise. By our priority definition, in $\left[t, t^{\prime}\right), J_{U}$ executes or suspends whenever $J_{L}$ executes (for $e_{L}^{\prime}$ time units). Therefore, after $t^{\prime}, J_{U}$ has at $\operatorname{most} \max \left(0, e_{U}^{\prime}+s_{U}^{\prime}-e_{L}^{\prime}\right)$ computation and suspension time left. Given that $J_{U U}$ has completed at $t, J_{U}$ is the only U-job of $J$ that may not have completed by time $t^{\prime}$. By Def. 3, job $J$ 's total idle blocking time is given by the unfinished computation and suspension time of $J$ 's U-jobs after $t^{\prime}$. Thus, $T_{i, v}^{k}$ 's total idle blocking time is at $\operatorname{most}^{2} \max \left(0, e_{U}^{\prime}+s_{U}-e_{L}^{\prime}\right) \leq e_{U}+s_{U}$, which equals $e_{i}^{k-1}+s_{i}^{k-1}$. Therefore, $T_{i, v}^{k}$,s total idle blocking time is at most $\sum_{j=1}^{j=k-1}\left(e_{i}^{j}+s_{i}^{j}\right)$.

\footnotetext{
${ }^{2}$ Since we do not assume that every job executes for its worst-case execution time, $J_{L}$ could execute for zero time units at run-time.
} 


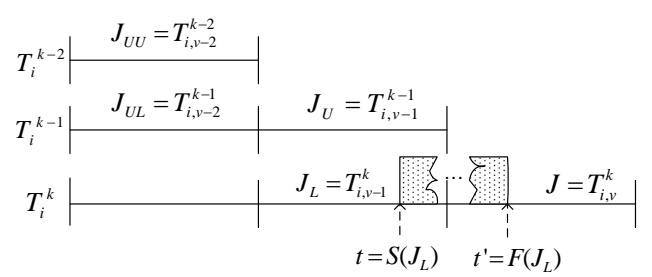

(a)

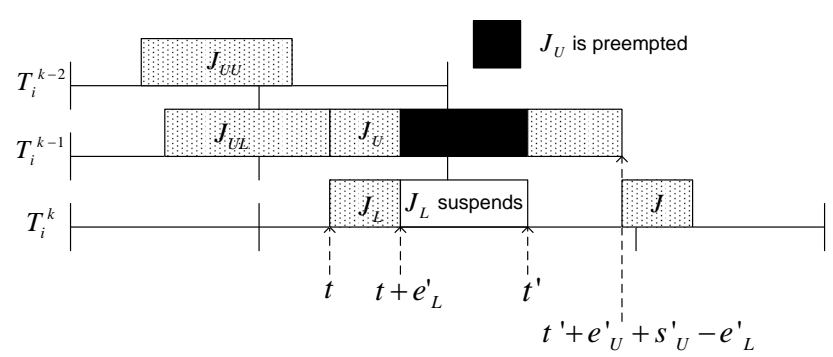

(b)

Figure 5: (a) Upper bounding the idle blocking time. (b) Case 1.

Case 2: $J_{U U}$ is not complete at $t$. Since $J_{U L}$ must have been complete at $t$ (otherwise, $J_{L}$ cannot start at $t$ ), $J_{U}$ is blocked at or after $t$. By the induction hypothesis, $J_{U}$ idle blocks for at most $\sum_{j=1}^{j=k-2}\left(e_{i}^{j}+s_{i}^{j}\right)$ time at or after $t$. Thus, $T_{i, v}^{k}$ 's total idle blocking time is at most the computation and suspension time left by $J$ 's U-jobs after $t$, which is at most $J_{U}$ 's total idle blocking time plus $J_{U}$ 's computation and suspension time, which is $\sum_{j=1}^{j=k-1}\left(e_{i}^{j}+s_{i}^{j}\right)$.

If $J_{L}$ or $J_{L}$ 's $\mathrm{U}$-jobs execute or suspend for less than their worst-case costs at run-time, then $J$ 's idle blocking time can increase, as illustrated in Fig. 6(a). In Case 1 of Lemma 2, we assumed that $J_{L}$ could execute for zero time units (see the footnote). And in Case 2 of Lemma 2, by applying the induction hypothesis to $J_{U}$, we are assuming that $J_{L}$ and all U-jobs of $J_{L}$ could execute for zero time units, in which case $J_{L}$ completes at the earliest possible time point and $J$ 's idle blocking time becomes maximal, as shown in Fig. 6(b). However, the proof in Lemma 2 is too pessimistic because it ignores the fact that if $J_{L}$ 's U-jobs execute for less at run-time, then the idle blocking time of $J_{L}$ will be decreased at the same time, as shown in Fig. 6(b). Lemma 4 deals with this pessimistism and minimizes the per-job idle blocking time needed to be treated as suspensions. Before proving Lemma 4, we first prove Lemma 3, which gives the latest possible enabled time of any tardy job $T_{i, v}^{k}$.

Definition 8. Let $\max _{k}=\max \{j \mid 1 \leq j \leq k \leq m \wedge$ $\left.\left(\forall w: 1 \leq w \leq k: e_{i}^{j}+s_{i}^{j} \geq e_{i}^{w}+s_{i}^{w}\right)\right\}$. That is, the maximum total execution cost and suspension length among the subtasks $\left\{T_{i}^{1}, T_{i}^{2}, \ldots, T_{i}^{k}\right\}$ is maximal for $T_{i}^{\max _{k}}$. If $k=$ 0 , then let $\max _{k}=1$.
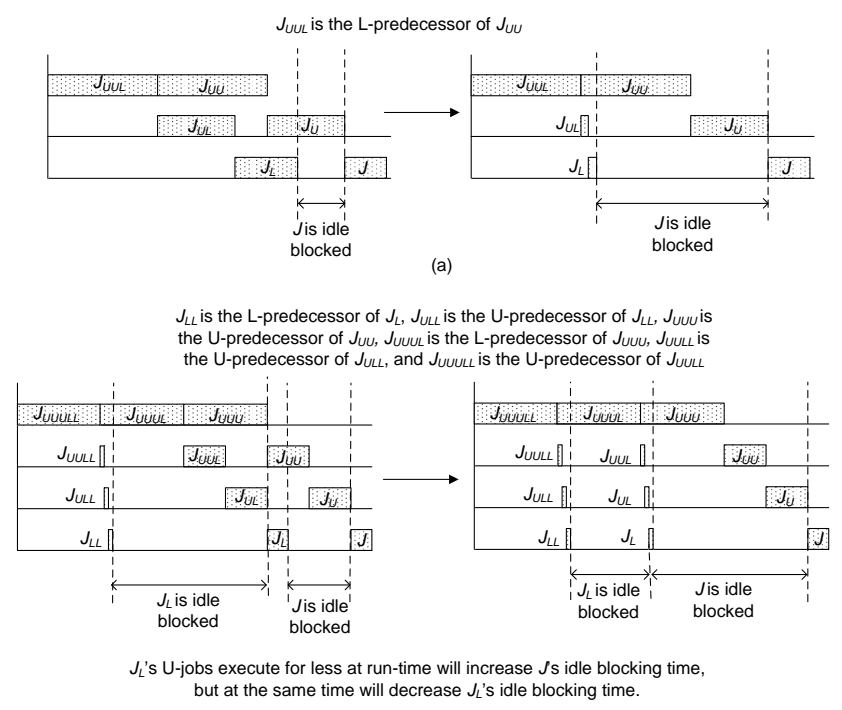

(b)

Figure 6: (a) $J$ 's idle blocking time can be increased if $J_{L}$ and $J_{L}$ 's U-jobs execute for less at run-time. (b) The idle blocking time of $J_{L}$ will be decreased if $J_{L}$ 's U-jobs execute for less at run-time.

Definition 9. Let $\Delta^{1}$ be the total length of all maximal subintervals within $\left[\max \left(r_{i, v-1}^{1}, F\left(T_{i, v-2}^{1}\right)\right), E T\left(T_{i, v+1}^{1}\right)\right)$ during which some $\mathrm{U}$-job of $T_{i, v+1}^{k}$ or $T_{i, v}^{k}$ is preempted. Let $\Delta^{j}$ (where $1<j \leq k$ ) be the total length of all maximal sub-intervals within $\left.\left[E T\left(T_{i, v+1}^{j-1}\right)\right), E T\left(T_{i, v+1}^{j}\right)\right)$ during which some $\mathrm{U}$-job of $T_{i, v+1}^{k}$ or $T_{i, v}^{k}$ is preempted. This is illustrated in Fig. 7.

Lemma 3. If $v \geq 3-k$ (recall that by adding "extra" initial jobs, $T_{i, 3-k}^{k}$ is the second job of $\left.T_{i}^{k}\right), k>$ 1 , and L3.1 and L3.2 below hold, then $\operatorname{ET}\left(T_{i, v+1}^{k}\right) \leq$ $\max \left(r_{i, v}^{1}, F\left(T_{i, v-1}^{1}\right)\right)+\sum_{j=1}^{k}\left(\Delta^{j}\right)+k \cdot\left(e_{i}^{\max _{k-1}}+\right.$

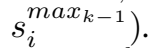

L3.1: $T_{i, v}^{k-1}$ and $T_{i, v+1}^{k-1}$ are both tardy.

L3.2: $T_{i, v}^{k}$ completes before $T_{i, v+1}^{k-1}$.

Proof. We prove the claim by induction on $k$. For the base case, $k=2$, we have $E T\left(T_{i, v+1}^{2}\right) \stackrel{L 3.1 \text { and }}{=} L 3.2$ $F\left(T_{i, v+1}^{1}\right) \leq E T\left(T_{i, v+1}^{1}\right)+\Delta^{2}+e_{i}^{1}+s_{i}^{1} \stackrel{L 3.1}{=} F\left(T_{i, v}^{1}\right)+$ $\Delta^{2}+e_{i}^{1}+s_{i}^{1} \leq \max \left(r_{i, v}^{1}, F\left(T_{i, v-1}^{1}\right)\right)+\Delta^{1}+e_{i}^{1}+s_{i}^{1}+$ $\Delta^{2}+e_{i}^{1}+s_{i}^{1} \leq \max \left(r_{i, v-1}^{1}, F\left(T_{i, v-2}^{1}\right)\right)+\Delta^{1}+\Delta^{2}+2$. $\left(e_{i}^{\max _{k-1}}+s_{i}^{\max _{k-1}}\right)$.

For the induction step, $k>2$, we have $E T\left(T_{i, v+1}^{k}\right) \quad \stackrel{L 3.1 \text { and }}{=} \operatorname{L3.2} F\left(T_{i, v+1}^{k-1}\right) \leq E T\left(T_{i, v+1}^{k-1}\right)+$ $\Delta^{k}+e_{i}^{k-1}+s_{i}^{k-1} \leq E T\left(T_{i, v+1}^{k-1}\right)+\Delta^{k}+e_{i}^{\max _{k-1}}+$ $s_{i}^{\max _{k-1}} \stackrel{\text { Ind. Hyp. }}{\leq} \max \left(r_{i, v-1}^{1}, F\left(T_{i, v-2}^{1}\right)\right)+\sum_{j=1}^{k-1} \Delta^{j}+$ $(k-1) \cdot\left(e_{i}^{\max _{k-2}}+s_{i}^{\max _{k-2}}\right)+\Delta^{k}+e_{i}^{\max _{k-1}}+s_{i}^{\max _{k-1}} \leq$ 


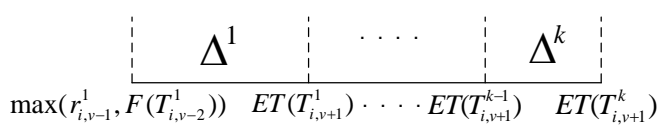

Figure 7: Def. 9. $\underset{\left.s_{i}^{\max _{k-1}}\right) .}{\left.\max _{i, v-1}, F\left(T_{i, v-2}^{1}\right)\right)+\sum_{j=1}^{k} \Delta^{j}+k \cdot\left(e_{i}^{\max _{k-1}}+\right.}+$

Lemma 4. The total idle blocking time ${ }^{3}$ of any two consecutive jobs $T_{i, v}^{k}$ and $T_{i, v+1}^{k}$ in $\tau^{P S}$ is at most $k \cdot\left(e_{i}^{\max _{k-1}}+\right.$

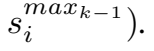

Proof. If $k=1$, then $T_{i, v}^{k}$ and $T_{i, v+1}^{k}$ cannot be idle blocked, so assume $k>1$. We prove the lemma in this case by induction on $v$.

If at most one of $T_{i, v}^{k}$ and $T_{i, v+1}^{k}$ is idle-blocked, then by Lemma 2 , the idle blocking time for both is at most $\sum_{j=1}^{j=k-1}\left(e_{i}^{j}+s_{i}^{j}\right)$, which is at most $k \cdot\left(e_{i}^{\max _{k-1}}+s_{i}^{\max _{k-1}}\right)$. This reasoning applies in the base case (where $v=2-k$ ) because $T_{i, 2-k}^{k}$ has no L-predecessor and thus is not idle blocked. In the rest of the proof, we consider the induction step, $v>2-k$, and assume that $T_{i, v}^{k}$ and $T_{i, v+1}^{k}$ are both idle blocked. By Def. 6, this implies that both $T_{i, v}^{k-1}$ and $T_{i, v+1}^{k-1}$ are tardy, and $T_{i, v}^{k}$ completes before $T_{i, v+1}^{k-1}$.

As shown in Fig. 8, by Def. 3, $T_{i, v}^{k}$ and $T_{i, v+1}^{k}$ can be PLblocked only in the interval $\left[E T\left(T_{i, v-1}^{k}\right), E T\left(T_{i, v+1}^{k}\right)\right)$. Given that $T_{i, v-1}^{1}$ is the first-stage U-job of $T_{i, v-1}^{k}$, $E T\left(T_{i, v-1}^{k}\right) \geq \max \left(r_{i, v-1}^{1}, F\left(T_{i, v-2}^{1}\right)\right) . \quad$ Thus, $T_{i, v}^{k}$ and $T_{i, v+1}^{k}$ can be PL-blocked only within $\left[\max \left(r_{i, v-1}^{1}, F\left(T_{i, v-2}^{1}\right)\right), E T\left(T_{i, v+1}^{k}\right)\right) . \quad$ By Def. 6, $T_{i, v}^{k} \quad$ (respectively, $T_{i, v+1}^{k}$ ) is idle blocked at $t \in\left[\max \left(r_{i, v-1}^{1}, F\left(T_{i, v-2}^{1}\right)\right), E T\left(T_{i, v+1}^{k}\right)\right)$ only if, at $t$, not all processors are occupied by jobs with higher priority than $T_{i, v}^{k}$ (respectively, $T_{i, v+1}^{k}$ ).

Thus, for any time $t \in\left[\max \left(r_{i, v-1}^{1}, F\left(T_{i, v-2}^{1}\right)\right)\right.$, $\left.E T\left(T_{i, v+1}^{k}\right)\right)$, if any U-job of $T_{i, v}^{k}$ or $T_{i, v+1}^{k}$ is preempted at $t$, then neither $T_{i, v}^{k}$ nor $T_{i, v+1}^{k}$ is idle blocked at $t$. This is because, by our priority definition, any U-job of $T_{i, v}^{k}$ or $T_{i, v+1}^{k}$ has higher priority than $T_{i, v}^{k}$ and $T_{i, v+1}^{k}$. Thus, all processors are occupied by jobs with higher priority than $T_{i, v}^{k}$ and $T_{i, v+1}^{k}$ at $t$. The total length of such time intervals within $\left[\max \left(r_{i, v-1}^{1}, F\left(T_{i, v-2}^{1}\right)\right), E T\left(T_{i, v+1}^{k}\right)\right)$, during which some $\mathrm{U}$-job of $T_{i, v}^{k}$ or $T_{i, v+1}^{k}$ is preempted, is given by $\sum_{j=1}^{k} \Delta^{j}$, by Def. 9 .

By Lemma 3 , the latest possible enabled time of $T_{i, v+1}^{k}$ is $E T\left(T_{i, v+1}^{k}\right) \leq \max \left(r_{i, v-1}^{1}, F\left(T_{i, v-2}^{1}\right)\right)+$ $\sum_{j=1}^{k} \Delta^{j}+k \cdot\left(e_{i}^{\max _{k-1}}+s_{i}^{\max _{k-1}}\right)$. Thus, the total idle blocking time of $T_{i, v}^{k}$ and $T_{i, v+1}^{k}$ is at most

\footnotetext{
${ }^{3}$ By this, we mean the sum of all maximal sub-intervals during which at least one of $T_{i, v}^{k}$ and $T_{i, v+1}^{k}$ is idle-blocked.
}

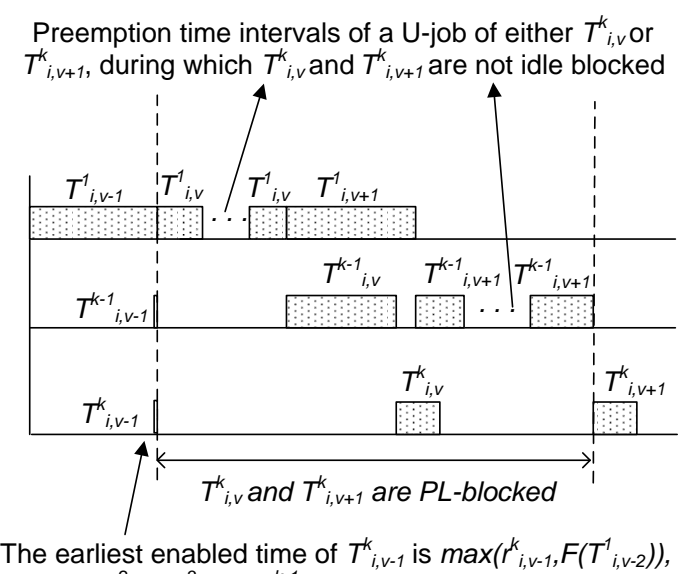

when $T_{i, v-1}^{2}, T_{i, v-1}^{3} \ldots T^{k-1}{ }_{i, v-1}$ execute for zero time unit

Figure 8: Lemma 4.

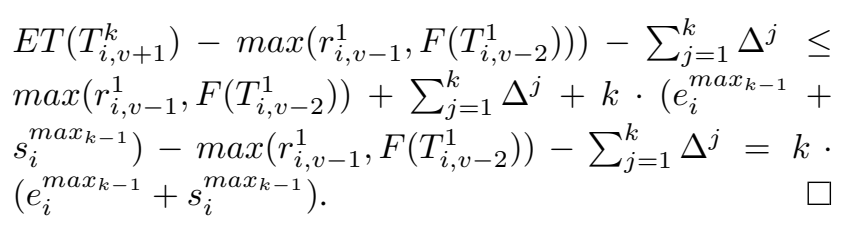

Thus, for any job $T_{i, v}^{k}$, if we increase $s_{i}^{k}$ by $\frac{k \cdot\left(e_{i}^{\max _{k-1}}+s_{i}^{\max _{k-1}}\right)}{2}$, then by Lemma 4, we are able to treat every job's idle blocking time as suspension time. Note that it might be necessary to treat $J$ 's idle blocking time as $J_{L}$ 's suspension time (this suspension time would be added to the end of $J_{L}$ 's execution instead of the beginning of $J$ 's). However, the total idle blocking time for any two consecutive jobs are upper-bounded by $k \cdot\left(e_{i}^{\max _{k-1}}+s_{i}^{\max _{k-1}}\right)$. In this way, we can transform each subtask $T_{l}^{h}$ to an independent suspending task. Note that we do not need to transform first-stage subtasks because they cannot be PL-blocked (see Def. 3). By transforming every subtask in $\tau^{P S}$ in this way, we obtain $\tau^{S}$.

Example 2. Consider a pipeline task $T_{1}$ with three stages, where $\left(e_{1}^{1}, s_{1}^{1}\right)=(1,1),\left(e_{1}^{2}, s_{1}^{2}\right)=(2,1)$, and $\left(e_{1}^{3}, s_{1}^{3}\right)=$ $(1,1) . T_{1}^{3}$ can be tramsformed into an independent suspending task by adding $\frac{k \cdot\left(e_{i}^{\max _{k-1}}+s_{i}^{\max _{k-1}}\right)}{2}=4.5$ time units to $s_{1}^{3}$, and $T_{1}^{2}$ can be transformed into an independent suspending task by adding $\frac{k \cdot\left(e_{i}^{\max _{k-1}}+s_{i}^{\max _{k-1}}\right)}{2}=3$ time units to $s_{1}^{2}$. Thus, this pipeline task can be transformed into three independent suspending tasks $T_{1}, T_{2}$, and $T_{3}$, where $\left(e_{1}, s_{1}\right)=(1,1),\left(e_{2}, s_{2}\right)=(2,4)$, and $\left(e_{3}, s_{3}\right)=(1,5.5)$.

Theorem 2. For any concrete instantiation $\bar{\tau}^{P S}$ of $\tau^{P S}$, there exists a concrete instantiation $\bar{\tau}^{S}$ of $\tau^{S}$ such that $\bar{\tau}^{P S}$ and $\bar{\tau}^{S}$ have equivalent GEDF schedules. 
Note again that this transformation strongly exploits the fact that, in our task model, suspension phases are upperbounded, and hence, can be reduced to reflect actual PLblocking times. Note also that the "reverse" of this theorem may not hold: for a concrete instantiation $\bar{\tau}^{S}$ of $\tau^{S}$, there may not exist a concrete instantiation $\bar{\tau}^{P S}$ of $\tau^{P S}$ such that $\bar{\tau}^{S}$ and $\bar{\tau}^{P S}$ have equivalent GEDF schedules.

Corollary 2. For any subtask $T_{l}^{h}$, if the maximum response time of any job of $T_{l}^{h}$ in any GEDF schedule for $\tau^{S}$ is z time units, then the maximum response time of any such job in any GEDF schedule for $\tau^{P S}$ is at most $z$ time units.

\section{Tardiness Analysis}

In this section, we first overview some recent results regarding tardiness in task systems with only suspensions [9], as stated in Theorem 3. Then we analyze the tardiness of NPS task systems by transforming such systems into systems with only suspensions.

\subsection{Tardiness Bound for Suspending Task Systems}

A suspending task system is just a special case of our system model as described in Sec. 2, where $b_{\max }=0$ and each task is a pipeline task consisting of only one subtask. For conciseness, we drop the superscript " 1 " as used in the pipeline task model when referring to a first-stage subtask, and simply use $T_{i, j}, r_{l, j}, d_{l, j}, e_{l}$, and $s_{l}$. We require $e_{l}+s_{l} \leq p_{l}$ and $U_{\text {sum }} \leq m$; otherwise, tardiness can grow unboundedly. A common case for real-time workloads is that both suspending tasks and computational tasks (which do not suspend) co-exist. To reflect this, we let $U_{\text {sum }}^{s}$ denote the total utilization of all suspending tasks, and $U_{\text {sum }}^{c}$ denote the total utilization of all computational tasks.

Definition 10. Let $s_{\max }=\max \left\{s_{1}, s_{2}, \ldots, s_{n}\right\}$. Let $\xi_{i}=$ $\frac{s_{\max }}{s_{\max }+e_{i}}$ be the suspension ratio of $T_{i}$. Let $\xi_{\max }=$ $\max \left\{\xi_{1}, \xi_{2}, \ldots, \xi_{n}\right\}$ be the maximum suspension ratio.

Definition 11. Let $E_{\text {sum }}^{s}$ be the total execution cost of all suspending tasks in $\tau^{S}$. Let $E_{\text {sum }}$ be the total execution cost of all tasks in $\tau^{S}$. Let $S_{\text {sum }}^{s}$ be the total suspension length of all tasks in $\tau^{S}$. Let $u_{\max }^{s}$ be the maximum utilization of any suspending task in $\tau^{S}$.

Definition 12. Let $U_{L}^{c}$ be the sum of the $\min (m-1, c)$ largest computational task utilizations, where $c$ is the number of computational tasks in $\tau^{S}$. Let $E_{L}^{c}$ be the sum of the $\min (m-1, c)$ largest computational task execution costs in $\tau^{S}$.

Definition 13. Let $V=E_{\text {sum }}^{s}+E_{L}^{c}+u_{\text {max }}^{s} \cdot S_{\text {sum }}^{s}+(m-$ 1) $e_{l}+m \cdot s_{l}+3 n \cdot s_{\max }$.
Theorem 3. [9] The tardiness of any task $T_{l}$ in $\tau^{S}$ scheduled under GEDF is at most $x+e_{l}+s_{l}$, where

$$
x \geq \frac{V}{\left(1-\xi_{\max }\right) \cdot m-U_{\text {sum }}^{s}-U_{L}^{c}},
$$

provided $U_{\text {sum }}^{s}+U_{L}^{c}<\left(1-\xi_{\max }\right) \cdot m$.

$x$ is well-defined provided $U_{\text {sum }}+U_{L}^{c}<\left(1-\xi_{\max }\right) \cdot m$. If this condition holds and $x$ equals the right-hand side of (1), then the tardiness of $T_{l, j}$ will not exceed $x+e_{l}+s_{l}$. A value for $x$ that is independent of the parameters of $T_{l}$ can be obtained by replacing $(m-1) e_{l}+m \cdot s_{l}$ with $\max _{l}((m-$ 1) $\left.e_{l}+m \cdot s_{l}\right)$ in $V$.

It is worth noting that this approach allows certain suspending tasks to be designated as computational tasks. In particular, all suspension phases of any task $T_{l}$ can be treated as computation phases, and $T_{l}$ can be considered to be a computational task with execution cost of $e_{l}+s_{l}$ time units and $u_{l}=\frac{e_{l}+s_{l}}{p_{l}}$.

\subsection{Tardiness Bound for NPS Task Sys- tems}

By transforming a periodic NPS task system into a periodic task system with only suspensions, we can apply Theorem 3 to the transformed task system and derive a tardiness bound for the NPS task system.

Definition 14. Ordinary tasks are those tasks in $\tau^{N P S}$ that do not contain suspensions, pipelines, and non-preemptive sections.

According to the transformations described in Secs. 3-5, for any subtask $T_{l}^{k}$ in $\tau^{N P S}$, we increase $s_{l}^{k}$ by $c_{l}^{k} \cdot b_{\max }$ (the maximum NP-blocking time as stated in Lemma 1). Then for any non-first-stage subtask $T_{l}^{h}$ (where $h \geq 2$ ), we increase $s_{l}^{h}$ by $\left(h \cdot\left(e_{l}^{\max _{h-1}}+s_{l}^{\max _{h-1}}\right)\right) / 2$ (to account for idle blocking times, which are bounded as in Lemma 4). Then every subtask in $\tau^{N P S}$ can be transformed into an independent task with only suspensions, which is mapped to a task in $\tau^{S}$ with the same task parameters. Since an ordinary task has only one computation phase, it can be NP-blocked for at most $b_{\max }$ time units. Thus, each ordinary task becomes a suspending task with a suspension length of $b_{\max }$ time units. Since in real systems most scenarios where nonpreemptivity arises are due to system calls and critical sections that are very short compared to job execution times, we can consider each ordinary task to be a computational task after the transformations by treating the suspension phase of any ordinary task $T_{l}$ as a computation phase. That is, $T_{i}$ is considered to be a computational task with execution cost of $e_{l}+b_{\max }$ time units and $u_{i}=\left(e_{i}+b_{\max }\right) / p_{i}$.

By Corollaries 1-2, any maximum per-subtask tardiness bound for $\tau^{S}$ holds for $\tau^{N P S}$ as well. After transforming $\tau^{N P S}$ into $\tau^{S}$, we can apply Theorem 3 to $\tau^{S}$ and obtain a tardiness bound for $\tau^{N P S}$, as stated in the following theorem. 
Theorem 4. The tardiness of any task $T_{l}$ in $\tau^{S}$ (and thus $\tau^{N P S}$ ) scheduled under GEDF is at most $x+e_{l}+s_{l}$, where $x$ is defined in Eq. (1), provided $U_{\text {sum }}^{s}+U_{L}^{c}<\left(1-\xi_{\max }\right) \cdot m$.

According to the suspending task model defined in Sec. 5.1, for any task $T_{l}$, it is required that $e_{l}+s_{l} \leq p_{l}$. Therefore, given that any subtask $T_{l}^{h}$ in $\tau^{N P S}$ is transformed into an independent suspending task by increasing its suspension length, in order to apply Theorem 4 to $\tau^{N P S}$, it is required that, for any subtask $T_{l}^{h}, c_{l}^{h} \cdot b_{\max }+e_{l}^{h}+s_{l}^{h}+(h$. $\left.\left(e_{l}^{\max _{l-1}}+s_{l}^{\max _{h-1}}\right)\right) / 2 \leq p_{l}$. For any ordinary task $T_{i}$, it is required that $e_{i}+b_{\max } \leq p_{i}$.

\section{Experimental Evaluation}

In this section, we describe experiments conducted using randomly-generated task sets to evaluate the applicability of the tardiness bound in Theorem 4. Our goal is to examine how restrictive the theorem's utilization cap is, for varying parameter choices.

Definition 15. A task set is said to be schedulable if it satisfies the utilization cap as stated in Theorem 4 and thus can have bounded tardiness.

Our methodology for generating random task sets was guided by practical considerations in two ways. First, we assume that non-preemptive sections are relatively short, for reasons discussed earlier. Second, we assume that for each pipeline, suspensions occur only in first- and last-stage subtasks. This is reflective of real-world scenarios in which input data is read in the first stage and output data is written in the last stage. With these considerations in mind, task sets were generated as follows. Task periods were uniformly distributed over $[200 \mathrm{~ms}, 300 \mathrm{~ms}] .{ }^{4}$ Subtask utilizations were uniformly distributed over $[0.001,0.3]$. Suspension lengths and non-preemptive section lengths were controlled by two parameters, $R_{S E}$ and $R_{N P E}$. $R_{S E}$ is defined as $S / E$, where $S$ is the suspension length of a subtask and $E$ is the execution cost of that subtask. $R_{S E}$ was varied as follows: 0.01 (suspensions are short), 0.05 (suspensions are moderate), and 0.1 (suspensions are long). The suspension-length ranges generated by these parameters can reasonably model the suspension lengths of real-world applications [9], as shown in Table 1. $R_{N P E}$ is defined as $b_{\max } / e_{\min }$, where $e_{\min }$ is the minimum execution cost among all subtasks. Given the above comments about non-preemptive sections being typically short, $R_{N P E}$ was set to be 0.01 in our experiments. To control the maximum idle-blocking time, a parameter $s_{\max }$, called pipeline stretch, was used, as defined below.

\footnotetext{
${ }^{4}$ Although we are using $1 \mathrm{~ms}$ as a time unit here, the definition of a time unit is flexible. For example, we could re-define a time unit to be $0.1 \mathrm{~ms}$, giving a period range of $[20 \mathrm{~ms}, 30 \mathrm{~ms}]$. Such a range might be more suitable for multimedia applications.
}

Table 1: Per-job suspension-length ranges.

\begin{tabular}{c|c|c|c|}
$\begin{array}{c}\text { suspension } \\
\text { length }\end{array}$ & $\begin{array}{c}\text { short } \\
\text { suspensions } \\
R_{S E}=0.01\end{array}$ & $\begin{array}{c}\text { moderate } \\
\text { suspensions } \\
R_{S E}=0.05\end{array}$ & $\begin{array}{c}\text { long } \\
\text { suspensions } \\
R_{S E}=0.1\end{array}$ \\
\hline min: & $2 \mu \mathrm{s}$ & $10 \mu \mathrm{s}$ & $20 \mu \mathrm{s}$ \\
avg: & $570 \mu \mathrm{s}$ & $3 \mathrm{~ms}$ & $5.6 \mathrm{~ms}$ \\
max: & $1.5 \mathrm{~ms}$ & $7.5 \mathrm{~ms}$ & $15 \mathrm{~ms}$ \\
\hline
\end{tabular}

Table 2: Average tardiness as computed via Theorem 4.

\begin{tabular}{c|c|c|c|}
$\begin{array}{c}\text { suspension } \\
\text { length }\end{array}$ & $\begin{array}{c}\text { short } \\
\text { suspensions } \\
R_{S E}=0.01\end{array}$ & $\begin{array}{c}\text { moderate } \\
\text { suspensions } \\
R_{S E}=0.05\end{array}$ & $\begin{array}{c}\text { long } \\
\text { suspensions } \\
R_{S E}=0.1\end{array}$ \\
\hline Tardiness & $167.5 \mathrm{~ms}$ & $404.8 \mathrm{~ms}$ & $824.2 \mathrm{~ms}$ \\
\hline
\end{tabular}

Definition 16. Let $s_{i}^{w}=\frac{e_{i}^{\max _{w}}+s_{i}^{\max _{w}}-e_{i}^{w}-s_{i}^{w}}{e_{i}^{\max _{w}}+s_{i}^{\max _{w}}}$. $s_{i}^{w}$ is called the subtask stretch of $T_{i}^{w}$. Let $s_{i}=$ $\max \left\{s_{i}^{1}, s_{i}^{2}, \ldots, s_{i}^{m}\right\} . s_{i}$ is called the task stretch of pipeline task $T_{i}$. Let $s_{\max }=\max \left\{s_{1}, s_{2}, \ldots, s_{n}\right\} . s_{\max }$ is called the pipeline stretch.

$s_{\max }$ was varied over $(0.01,0.05,0.1,0.15,0.2,0.25$, $0.3)$. A larger value of $s_{\max }$ indicates a larger value of the maximum idle blocking time (as stated in Lemma 2), which negatively impacts schedulability. We also varied $U_{\text {sum }}$ within $\{1,2,3,4,5,6,7,8\}$ and let $90 \%$ of the tasks in each task set be ordinary tasks. For each combination of $\left(R_{S E}, s_{\text {max }}, U_{\text {sum }}\right), 1,000$ task sets were generated for an eight-processor system.

The schedulability results that were obtained are shown in Fig. 9. Each column in this figure shows the percentage of the generated task sets for that set of parameter choices that were deemed to be schedulable. In general, very high schedulability can be achieved when $R_{S E}$ and $s_{\max }$ are kept small. For instance, when $R_{S E}=0.01$ and $s_{\max } \leq 0.05$, almost $100 \%$ of all task sets are schedulable if the system is not heavily-utilized, as shown in Fig. 9 (a). Moreover, high schedulability can be achieved when $s_{\max }$ is kept small and the system is moderately loaded. For instance, when $s_{\max } \leq 0.05$ and $U_{\text {sum }} \leq 4$, around $100 \%$ (respectively, $90 \% / 70 \%$ ) of all task sets are schedulable when suspensions are short (respectively, moderate/long). On the other hand, our analysis is negatively impacted by increasing any one of these three parameters. Given that the utilization constraint stated in Theorem 3 depends crucially on the maximum suspension length and the total system utilization, increasing any one of these parameters in the original NPS task system will either increase the suspension length of the corresponding tasks in the transformed system or increase the total utilization, thus further increasing the possibility for a task set to violate the utilization constraint.

In addition to schedulability, the magnitude of tardiness, 


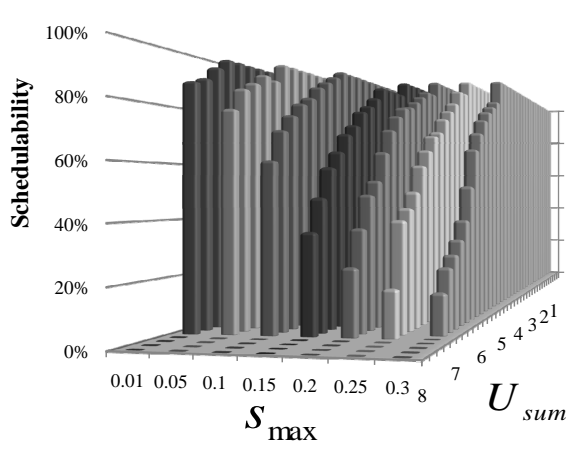

(a)

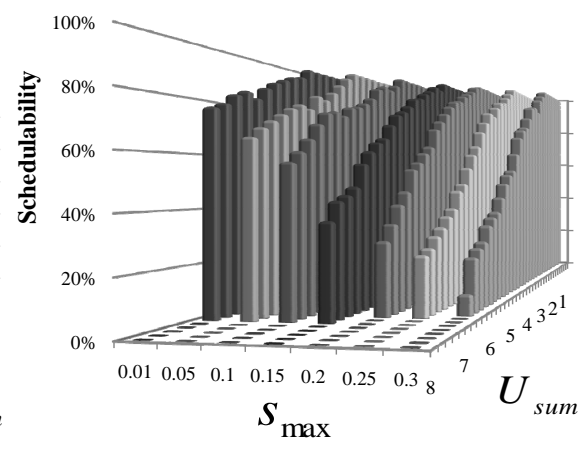

(b)

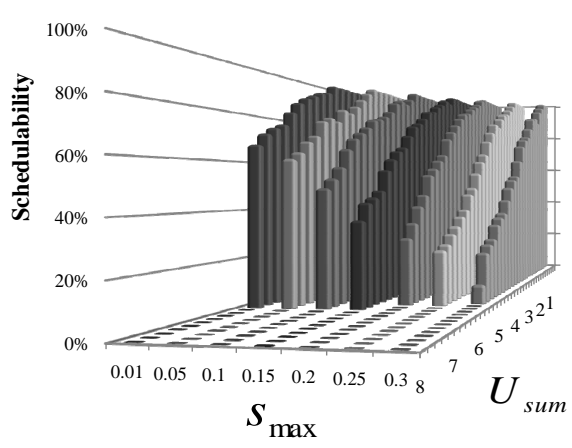

(c)

Figure 9: Schedulability results. (a) Short suspensions $\left(R_{S E}=0.01\right)$. (b) Moderate suspensions $\left(R_{S E}=0.05\right)$. (c) Long suspensions $\left(R_{S E}=0.1\right)$. Each figure gives a 3-D graph in which schedulability is shown under different combinations of two parameters: $s_{\max }$ and $U_{\text {sum }}$.

as computed using Theorem 4, is of importance. Table. 2 depicts the average of the computed bounds for each of the tested scenarios in our experimental framework for the case where $U_{\text {sum }}=4$ and $s_{\max }=0.05$ (that is, for each scenario in this case, an average of all bounds for all tasks in all schedulable task sets is given). As can be seen, tardiness is reasonable if the total utilization is moderate and suspensions are short or moderate. However, as suspension lengths increase, tardiness increases, as an examination of the bound in Theorem 4 suggests should be the case.

\section{Conclusion}

In this paper, we presented a method for transforming a periodic NPS task system into a simpler periodic task system with only suspensions. The transformation allows maximum response-time bounds derived for periodic suspending task systems to be applied to periodic NPS task systems. This allowed us to derive a deadline tardiness bound under GEDF for such systems. This bound shows that NPS task systems can be supported with bounded tardiness provided certain utilization constraints are met.

\section{References}

[1] U. Devi. An improved schedulability test for uniprocessor periodic task systems. In Proc. of the 15th Euromicro Conf. on Real-Time Systems, pp. 23-30, 2003.

[2] U. C. Devi and J. H. Anderson. Tardiness bounds under global EDF scheduling on a multiprocessor. In Proc. of the 26th IEEE Int'l Real-Time Systems Symp., pp. 330-341, 2005.

[3] P. Jayachandran and T. Abdelzaher. Transforming distributed acyclic systems into equivalent uniprocessors under preemptive and non-preemptive scheduling. In Proc. of the 20th Euromicro Conf. on Real-Time Systems, pp. 233-242, 2008.

[4] P. Jayachandran and T. Abdelzaher. A delay composition theorem for real-time pipelines. In Proc. of the 19th Euromicro Conf. on Real-Time Systems, pp. 29-38, 2007.
[5] I. Kim, K. Choi, S. Park, D. Kim, and M. Hong. Real-time scheduling of tasks that contain the external blocking intervals. In Proc. of the 2nd Int'l Workshop on Real-Time Computing Systems and Applications, pp. 54-59, 1995.

[6] H. Leontyev and J. H. Anderson. Generalized tardiness bounds for global multiprocessor scheduling. In Proc. of the 28th Real-Time Systems Symp., pp. 413-422, 2007.

[7] C. Liu and J. H. Anderson. Supporting pipelines in soft realtime multiprocessor systems. In Proc. of the $21^{\text {st }}$ Euromicro Conf. on Real-Time Systems, pp. 269-278, 2009.

[8] C. Liu and J. H. Anderson. Supporting sporadic pipelined tasks with early-releasing in soft real-time multiprocessor systems. In Proc. of the 15th IEEE Int'l Conf. on Embedded and Real-Time Computing Systems and Applications, pp. 284-293, 2009.

[9] C. Liu and J. H. Anderson. Task scheduling with selfsuspensions in soft real-time multiprocessor systems. In Proc. of the 30th Real-Time Systems Symp., pp. 425-436, 2009.

[10] J. W. S. Liu. Real-Time Systems. Prentice Hall, 2000.

[11] J. C. Palencia and M. Gonzlez Harbour. Schedulability analysis for tasks with static and dynamic offsets. In Proc. of the 19th IEEE Real-Time Systems Symp., pp. 26-37, 1998.

[12] J. C. Palencia and M. Gonzlez Harbour. Response time analysis of EDF distributed real-time systems. In J. Embedded Comput., Vol.1, pp. 225-237, 2005.

[13] R. Pellizzoni and G. Lipari. Improved schedulability analysis of real-time transactions with earliest deadline scheduling. In Proc. of the 11th IEEE Int'l Real Time and Embedded Technology and Applications Symp., pp. 66-75, 2005.

[14] R. Rajkumar. Dealing with Suspending Periodic Tasks. IBM Thomas J. Watson Research Center, 1991.

[15] F. Ridouard and P. Richard. Worst-case analysis of feasibility tests for self-suspending tasks. In Proc. of the 14th Real-Time and Network Systems, pp. 15-24, 2006.

[16] F. Ridouard, P. Richard, and F. Cottet. Negative results for scheduling independent hard real-time tasks with selfsuspensions. In Proc. of the 25th IEEE Real-Time Systems Symp., pp. 47-56, 2004. 